\title{
Fluctuation analysis of solar radio bursts associated with geoeffective X-class flares ${ }^{2 / 2}$
}

\author{
T.B. Veronese ${ }^{\mathrm{a}, *}$, R.R. Rosa ${ }^{\text {a }}$, M.J.A. Bolzan ${ }^{\text {b }}$, F.C. Rocha Fernandes ${ }^{c}$, H.S. Sawant ${ }^{\mathrm{d}}$, M. Karlicky ${ }^{\mathrm{e}}$ \\ a Lab for Computing and Applied Mathematics, Instituto Nacional de Pesquisas Espaciais (INPE), Brazil

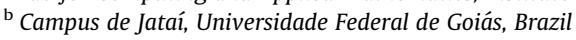 \\ ' IPE'D, Universidade do Vale do Paraíba, Brazil \\ d Astrophysics Division, INPE, Brazil \\ e Astronomical Institute of the Academy of Sciences of the Czech Republic, Czech Republic
}

\section{A R T I C L E I N F O}

\section{Article history:}

Received 1 April 2010

Received in revised form

4 September 2010

Accepted 27 September 2010

\section{Keywords:}

Decimetric solar radio bursts

Solar flares

Detrended fluctuation analysis

Computational data analysis

Space weather

\begin{abstract}
A B S T R A C T
High temporal resolution solar observations in the decimetric range $(1-3 \mathrm{GHz})$ can provide additional information on solar active regions dynamics and thus contribute to better understanding of solar geoeffective events as flares and coronal mass ejections. The June 6, 2000 flares are a set of remarkable geoeffective eruptive phenomena observed as solar radio bursts (SRB) by means of the $3 \mathrm{GHz}$ Ondrejov Observatory radiometer. We have selected and analyzed, applying detrended fluctuation analysis (DFA), three decimetric bursts associated to X1.1, X1.2 and X2.3 flare-classes, respectively. The association with geomagnetic activity is also reported. DFA method is performed in the framework of a radio burst automatic monitoring system. Our results may characterize the SRB evolution, computing the DFA scaling exponent, scanning the SRB time series by a short windowing before the extreme event. For the first time, the importance of DFA in the context of SRB monitoring analysis is presented.
\end{abstract}

(c) 2010 Elsevier Ltd. All rights reserved.

\section{Introduction}

Solar space weather events like coronal mass ejections and solar flares are usually accompanied by solar radio bursts, which can be used for a low-cost real-time space weather monitoring (Lobzin et al., 2009). However, most of the solar radio flare signals are characterized by complex variability patterns including both non-stationarities and non-linearities. In fact, radio emission from solar active region electron beams can provide information about the non-linear electron acceleration/injection processes and the properties of the complex ambient coronal structures (Nindos et al., 2008). In particular, solar radio emissions in the decimetric frequency range (above $1 \mathrm{GHz}$ ) are very rich in temporal and spectral fine structures due to non-linear processes occurring in the magnetic structures on the corresponding active regions (Aschwanden et al., 1995). The importance of this decimetric solar flare scenario has been investigated, for example, from non-linear analysis of decimetric bursts at $3 \mathrm{GHz}$ observed during the June 6, 2000 flare (Rosa et al., 2008). It was found that the $3 \mathrm{GHz}$ radio burst power spectrum exhibits a power-law which is an evidence of stochastic intermittency due to a self-affine dynamics as found in the MHD turbulence theory. Intermittent

\footnotetext{
Funded by BZG.

* Corresponding author.

E-mail address: thalitabv@gmail.com (T.B. Veronese).
}

energetic process implies that the fluctuations are multi-scaling correlated as predicted in the models for multi-loop interactions (Tajima et al., 1987).

A common practice to characterize decimetric solar radio bursts signals is to record the digital dynamical spectra, extract sequences of single frequency events with limited discrete length (presented as a SFU time series) and to determine quantitative characteristics on the basis of these observations. From a solar radio flare monitoring perspective, the basic strategy is to determine appropriate characteristics of the signal that could be preserved in short temporal moving windowing used to scanning the whole data in a real time procedure providing a radio burst automatic monitoring system (RBAMS). Nowadays, decimetric solar bursts are observed with time resolution ranging from $10^{-1}$ to $10^{-3} \mathrm{~s}$. Solar radio flare signals are events ranging from 1 to 10 min long (including the pre and post-flare typical intervals), so that a typical decimetric solar burst time series is composed of $N$ measures ranging from the order of $10^{3}$ to $10^{4}$ measurement points. As power spectra index (from $1 / f^{\beta}$ power-laws), obtained from fast Fourier transform (FFT) of the signal auto-correlation function are robust only for $N \gg 10^{3}$, it fails in such monitoring characterization because of relatively short data records for decimetric solar radio bursts which contain stochastic components and non-stationarities.

In this paper it is shown that typical decimetric bursts frequency estimates, obtained from their Fourier-based spectral peaks, are affected by the window length and phase of signal component, thus presenting a large variance and low performance as a candidate 
measurement for decimetric solar RBAMS. Alternatively, it is shown that the method of detrended fluctuation analysis (DFA) (Peng et al., 1994) provides more robust measurements for monitoring solar decimetric bursts.

\section{Solar radio burst data}

The June 6, solar filament eruption was accompanied by intense solar flares. A type II radio burst and powerful series of solar eruptions including a full-halo coronal mass ejection (CME) were reported in association with this event (Solar Flare NOAA Report). The June 6, 2000 flares, classified as X1.1, X1.2 and X2.3, respectively, were observed during 13:36-17:00 UT in the active region NOAA AR 9026 (N21, E23). Images of the two main flares X1.2 and X2.3 were observed by the EIT/ SOHO and SXT/Yohkoh instruments (Rosa et al., 2005). The CME activity, associated with the flares in AR 9026, started to increase 17:30 UT reaching its maximum at 19:42 UT. Geomagnetic activity increased on June 8 to major geomagnetic storm conditions, especially at higher latitudes. The maximum intensity for Kyoto University's real-time Dst index ( $-90 \mathrm{nT})$ was recorded at 15:30 UT June 8 .

Fig. 1a shows the NOAA GOES 8 satellite X-ray flux (from channels: 0.5-4.0 A signal under 1.0-8.0 A signal). Three respective SRB were observed employing the Ondrejov $3 \mathrm{GHz}$ radio-spectrograph with time resolution of $0.01 \mathrm{~s}$ (Jiricka et al., 1993). The SRB1 was recorded with lower time resolution, $\tau=1.2 \mathrm{~s}$, from starting time $t_{0}=13: 28: 00$ UT to 13:40:00 UT representing a time series composed of $N=600$ digital measurements. The SRB1 duration $\Delta t_{d}$ was of $12 \mathrm{~min}$. The SRB2 was recorded with higher time resolution, $\tau=0.02 \mathrm{~s}$, from starting time $t_{0}=15: 04: 00$ UT to $15: 41: 40 \mathrm{UT}$, representing a time series composed of $N=125 \times 10^{3}$ digital measurements with duration $\Delta t_{d}=42 \mathrm{~min}$. The SRB3 was recorded with the highest time resolution, $\tau=0.01 \mathrm{~s}$, from starting time $t_{0}=16: 34: 50 \mathrm{UT}$ to $16: 35: 30 \mathrm{UT}$, representing a time series composed of $N=4000$ digital measurements (then, duration $\Delta t_{d}=40 \mathrm{~s}$ ). The basic characteristics of the selected SRB are summarized in Table 1 , where the time interval before the respective flares and CME peak are reported as $\Delta t_{b F}$ (column 7th) and $\Delta t_{b C M E}$ (column 8th), respectively. In Table 1 are shown also the windowing intervals $\Delta t_{w}\left(N_{w}\right)\left(\Delta t_{w}\right.$ is the time-lag while $N_{w}$ is the correspondent number of samples) which will be considered in our DFA analysis in Section 4.1

\section{Detrended fluctuation analysis method}

Detrended fluctuation analysis (DFA) measures scaling exponents from non-stationary time series for determining the statistical self-affinity of an underlying dynamical non-linear process. It is useful for characterizing temporal patterns that appear to be due to long-range memory stochastic processes. DFA has been used in several non-stationary time series analysis from biological and physiological data to finance and space physics signals (Bunde et al., 2000; Iyengar et al.,1996; Buldyrev et al., 1995; Bai and Zhu, 2010; Alvarez-Ramirez et al., 2009; Moret et al., 2003).

\subsection{DFA algorithm}

Detrending methods for fluctuation analysis have been recently proposed and applied for detection of persistent correlations in non-stationary time series analysis (Bashan et al., 2008). The DFA algorithm considered in our approach, introduced by Peng et al. (1994), is composed of six computational operations starting on a discrete time series of amplitudes $\left\{A_{i}\right\}$ :

- Discrete integration: Calculate the cumulative representation of $\left\{A_{i}\right\}$ as

$C(k)=\sum_{i=1}^{k}\left(A_{i}-\langle A\rangle\right), \quad(k=1,2, \ldots, N)$

where $\langle A\rangle=\sum_{i=1}^{N} A$ is the average of $\left\{A_{i}\right\}$.

- Windowing: Using an arbitrary local window of length $n$, divide $C(k)$ into non-overlapping $N_{n}=\operatorname{int}(N / n)$ sub-interval $c_{j}(j=1$, $\left.2, \ldots, N_{n}\right)$. Note that each sub-interval $c_{j}$ has length $n$ and $N$ may not be the integer multiple of $n$. Then, the series $C(k)$ is divided once more from the opposite side to make sure all
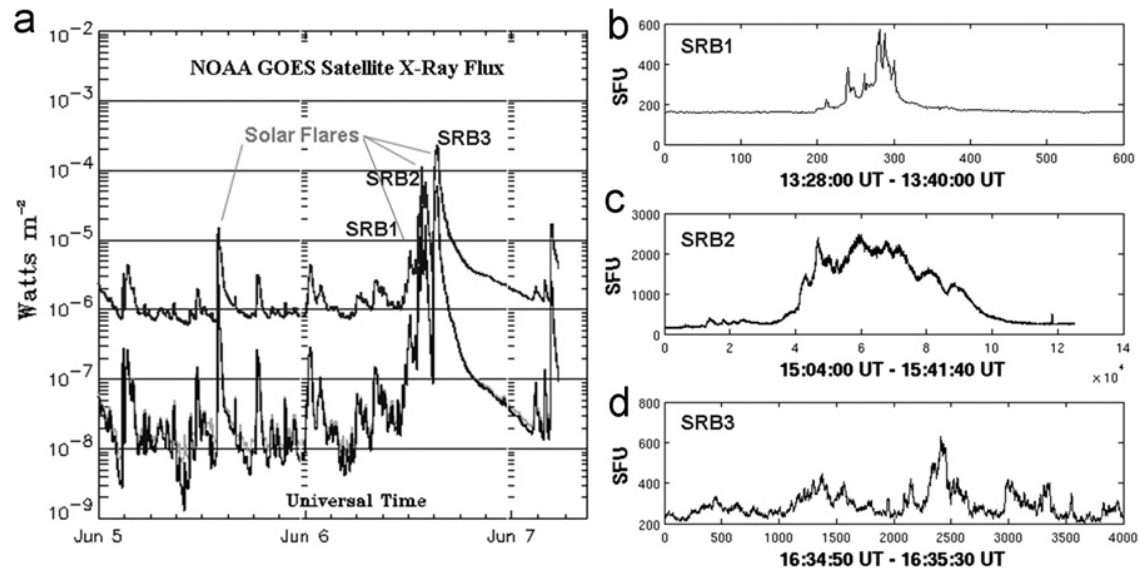

Fig. 1. (a) Solar flares and SRB identification on the X-ray flux in the 1.0-8.0 ^ band as measured by the NOAA GOES-8 satellite, (b) SRB1, (c) SRB2 and (d) SRB3.

Table 1

The basic characteristics of the June 6, 2000 SRBs observed at $3 \mathrm{GHz}$

\begin{tabular}{|c|c|c|c|c|c|c|c|}
\hline Event & $\tau(\mathrm{s})$ & $t_{0}(\mathrm{UT})$ & $\Delta t_{d}(N)$ & $\Delta t_{w}\left(N_{w}\right)$ & Class flare (UT) & $\Delta t_{b F}$ & $\Delta t_{b C M E}$ \\
\hline SRB1 & 1.2 & $13: 28: 00$ & $12 \min (600)$ & $1.2 \min (60)$ & X1.1 (13:36:00) & $8 \mathrm{~min}$ & $6 \mathrm{~h} 14 \mathrm{~min}$ \\
\hline SRB2 & 0.02 & $15: 04: 00$ & $\approx 42 \min \left(125 \times 10^{3}\right)$ & $4.2 \min (12.500)$ & $X 1.2(15: 36: 00)$ & $32 \mathrm{~min}$ & $4 \mathrm{~h} 38 \mathrm{~min}$ \\
\hline SRB3 & 0.01 & $16: 34: 50$ & $40 \mathrm{~s}(4000)$ & $4 \mathrm{~s}(400)$ & X2.3 (16:35:22) & $32 \mathrm{~s}$ & $3 \mathrm{~h} 08 \mathrm{~min}$ \\
\hline
\end{tabular}


points are addressed, performing at the end of this operation $2 N_{n}$ sub-intervals.

- Fitting: Get, in each sub-interval, the least-square fits as follows:

$p_{j}^{m}(k)=b_{j_{0}}+b_{j_{1}} k+\cdots+b_{j_{m-1}} k^{m-1}+b_{j_{m}} k^{m}, \quad m=1,2, \ldots$

where $m$ is interpreted as the order of the detrended trend, denoted here as $D F A^{m}$.

- Variance: Compute the cumulative deviation series in every subinterval, where the trend has been subtracted: $C_{j}(k)=C(k)-$ $p_{j}^{m}(k)$. Then, calculate the variance of the $2 N_{n}$ sub-intervals:

$F^{2}(j, n)=\left\langle C_{j}^{2}(i)\right\rangle=\frac{1}{n} \sum_{i=1}^{n}\left[C((j-1) n+i)-p_{j}^{m}(i)\right]^{2}$

for $j=1,2, \ldots, N_{n}$, and

$F^{2}(j, n)=\left\langle C_{j}^{2}(i)\right\rangle=\frac{1}{n} \sum_{i=1}^{n}\left[C\left(N-\left(j-N_{n}\right) n+i\right)-p_{j}^{m}(i)\right]^{2}$

for $j=N_{n}+1, N_{n}+2, \ldots, 2 N_{n}$.

- Fluctuation: Calculate the average of all the variances and the square root to get the fluctuation function of DFA $F(n)$ :

$F(n)=\left[\frac{1}{2 N_{n}} \sum_{j=1}^{2 N_{n}} F^{2}(j, n)\right]^{1 / 2}$

- Scaling exponent: Perform again, recursively, computation from windowing to calculation of corresponding $F(n)$ with different $n([N / 4]>n \geq 2 m+2)$ box lengths. In general, in the presence of fluctuations in the form of power law: $F(n)=K n^{\alpha}, F(n)$ increases linearly with increasing $n$. Then, using the linear least-square regression on the double $\log$ plot $\log F(n)=\log K+\alpha \log n$ one can get the slope $\alpha$, which is the scaling exponent of the DFA method.

\section{2. $P S D$ and DFA}

If $A\left(t_{k}\right)$ is the $k$-th value of a time series composed by $N$ discrete samples with time resolution $\tau$, its energy is given by
$E(k)=\sum_{0}^{N-1}\left|A\left(t_{k}\right)\right|^{2} \tau$ (for stationary stochastic process of infinite duration, energy is usually infinite) and power of the signal is defined as $P(k)=E(k) / N \tau$. Note that the units of $P$ are the square of the units of the time series and for zero-mean time series the power $P$ is equal to the variance of $\left\{A\left(t_{k}\right)\right\}$ with $i=1, \ldots, N$. Then, the distribution of $P$ (or variance) of a time series with frequency $1 / T$ (in Hertz, with $T=\tau+\Delta t$ ) is the so-called power spectral density (PSD) (Kay and Marple, 1981). In practical terms, admitting all possible frequencies from all possible scales $\Delta t$, PSD is the squared modulus of the Fourier transform of the rescaled time series and can be estimated based on the direct computation through FFT. In our analysis unit of PSD is (s.f.u $)^{2} / \mathrm{Hz}$.

The correlation function $C(\Delta t)$ decays with an exponent $C(\Delta t) \approx(\Delta t)^{-\gamma}$ and the PSD decays as $P(f) \approx(f)^{-\beta}$, where $f=1 / \Delta t$. Thus, the slope of the power spectrum $\beta$ is usually used to characterize different stochastic processes which are responsible for the autocorrelation range in a given time series. Based on the Wiener-Khinchin theorem (Kay and Marple, 1981), it is possible to show that the two exponents $\beta$ (from PSD) and $\alpha$ (from DFA) are related by $\beta=2 \alpha-1$. For fractional Brownian motion (a cumulative sum of fractional Gaussian noise) we have $1 \leq \beta \leq 3$, and then $1 \leq \alpha \leq 2$. The diagnostic potential of PSD and DFA methods is tested on a sub-set of Brownian noise time series. The canonical Brownian noise $(\mathrm{fBm})$ proxy time series was generated from the stochastic system as given by Osborne and Provenzale (1989) (see Rosa et al., 2008 for more technical details). A typical canonical Brownian noise, as shown in Fig. 2a, can be characterized by $\beta \approx 2$ and $\alpha \approx 1.5$. The fBm data were originally generated having $2 \times 10^{4}$ samples (Fig. 2a). This signal was sequentially splitted into 5 subseries containing $10^{4}, 5 \times 10^{3}, 10^{3}, 5 \times 10^{2}$, and 50 samples. Without loss of generality are shown in Figs. $2 b$ and $c$ the PSD and DFA for the original $2 \times 10^{4}$ series and for the sub-set containing the shortest number of samples $(N=50)$. Figs. $2 b, c, d$ and e show that values of $\beta$ are much less robust than values of $\alpha$ for short time series with $N \ll 10^{4}$. Fig. 2 f shows, in a double log plot, both the behaviors of $\beta$ and $\alpha$ versus the sub-series size. While the maximum deviation of $\alpha$ from the expected value is around $8 \%$, the
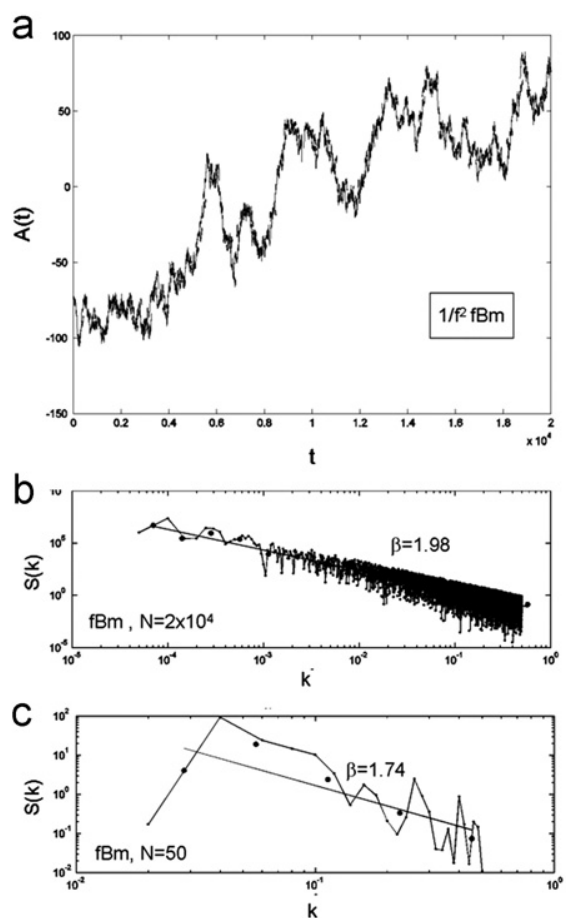

d
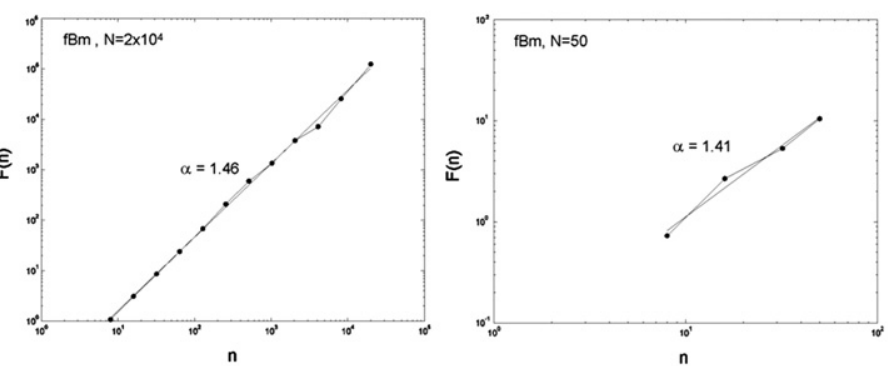

f

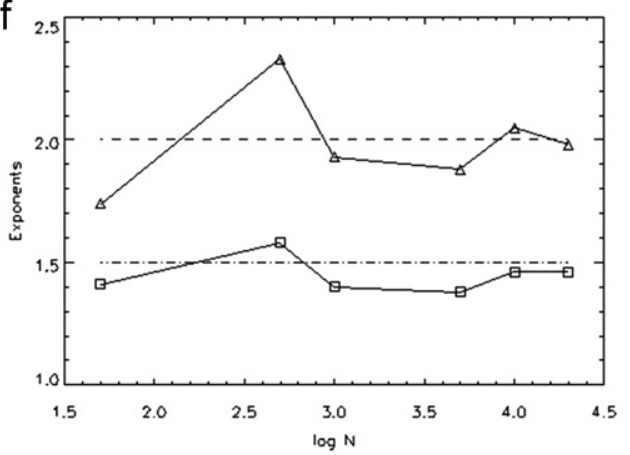

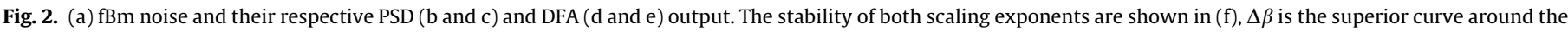
expected value $\beta=2$. 
same deviation for $\beta$ is more than $30 \%$. Then, the use of PSD fails for SRB monitoring because of relatively short data records (as SRB 1 and SRB 3). Fig. 2d shows that, for short time series, DFA can detect the correlation length more accurately than the PSD scaling exponent. Our results show that DFA method is especially useful for short records of stochastic and non-linear processes.

Other methods have been explored to analyze time profiles of solar radio burst - e.g. Watari (1996) and Zaitev et al. (2003). Even though these methods apprehend some important statistical or geometrical measurement from the data, they present, as PSD, a high bias when it is computed on short time series. It is worthy of note that short time series appear in our context due to the monitoring strategy, which is based on a possible real-time analysis application. The windowing in our approach mimics the real-time analysis in a real monitoring situation. Using fractal dimensions, for example, is out of question for monitoring purposes. The computation of fractal and/or correlation dimensions from 1D time series requires the high computational cost of phase space reconstruction technique (even for box-counting algorithm), whose first condition is to have a stationary and long time series ( $\rightarrow 10^{3}$ points) (Rempel et al., 2004). The data for the current monitoring analysis are non-stationary (due to turbulent process) and short (due to the monitoring window).

\section{Analysis of $3 \mathrm{GHz}$ SRB}

\subsection{Global scaling exponents}

With the DFA method, we analyze the fluctuations of each $3 \mathrm{GHz}$ SRB. From Figs. 3a-c, it is shown that $1<\alpha<2$ characterizes longrange correlations, non-stationary, multi-fractal random walk like and unbounded free-scaling process as predicted from the inhomogeneous multi-looping interaction on the solar active regions. However, the long-range characteristic implies, in the practical sense of using $\alpha$ as a monitoring measurement, that all the SRB can be analyzed locally for monitoring purposes.

Next we focus on the monitoring local analysis of each burst taking into consideration the time windowing before the peak of each correspondent flare, as shown in Figs. 3a-c.
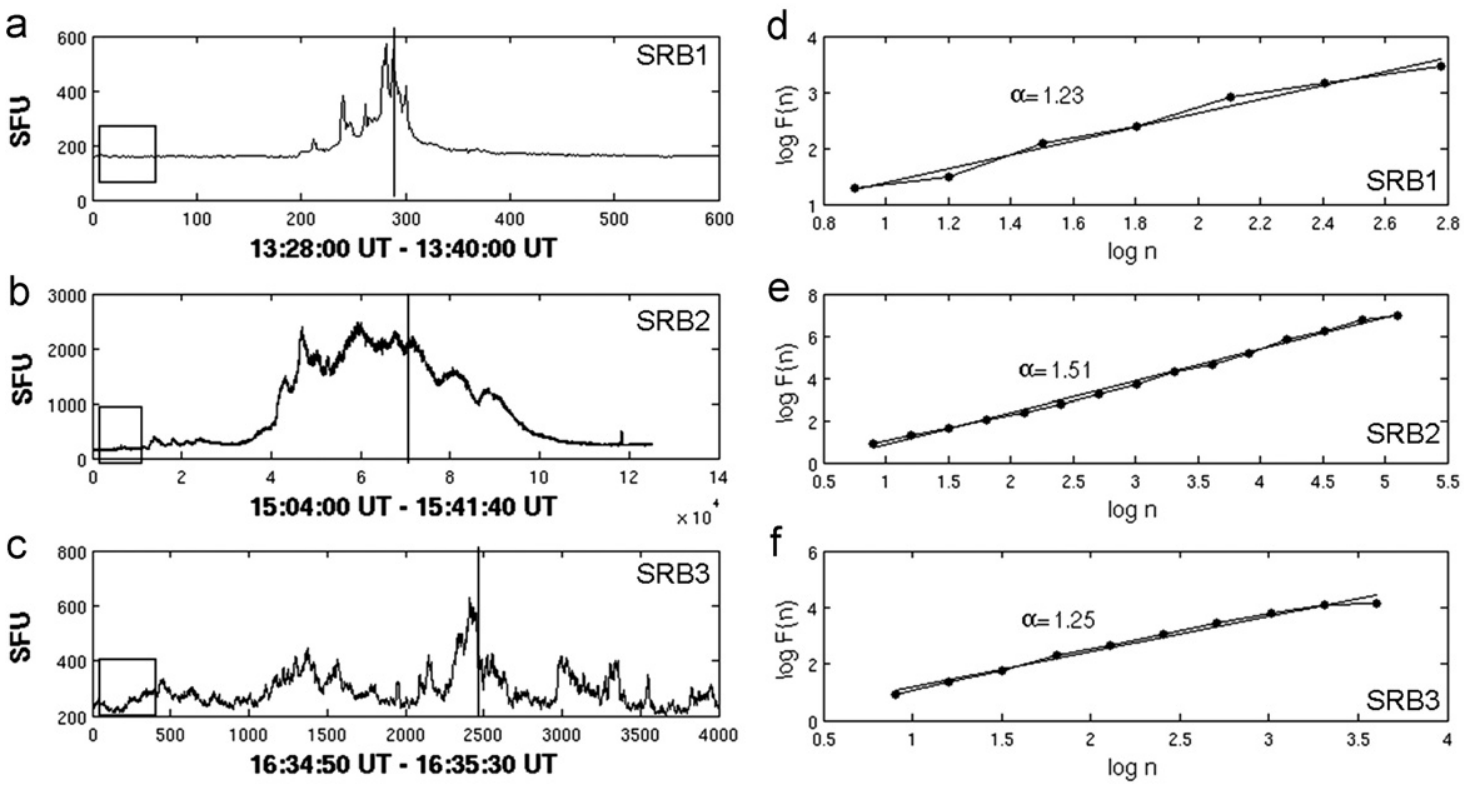

Fig. 3. The time profile of SRB1 (a), SRB2 (b) and SRB3 (c) showing the respective DFA scaling exponents in (d), (e) and (f). 
a
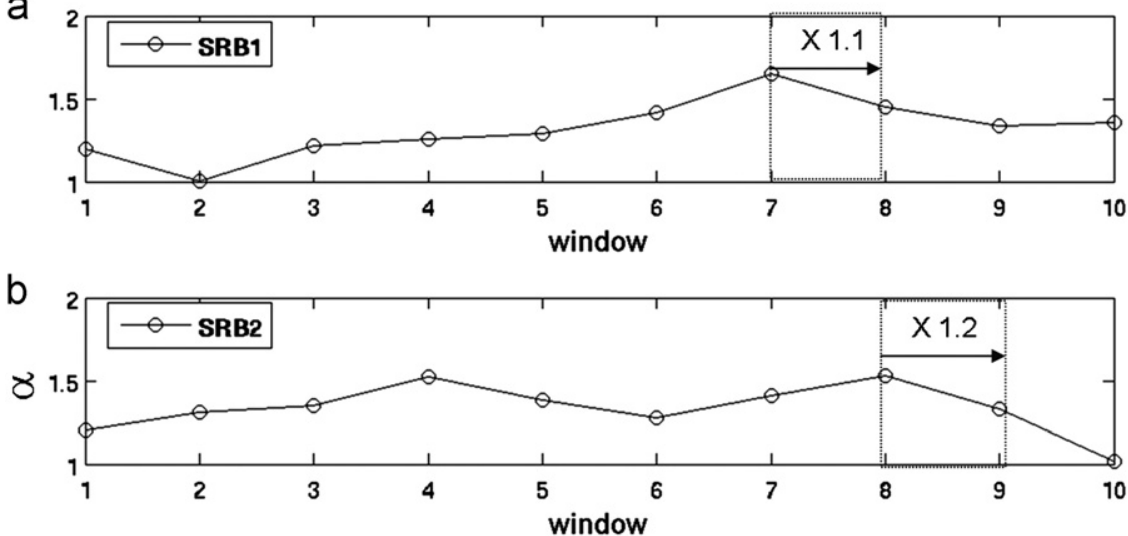

C

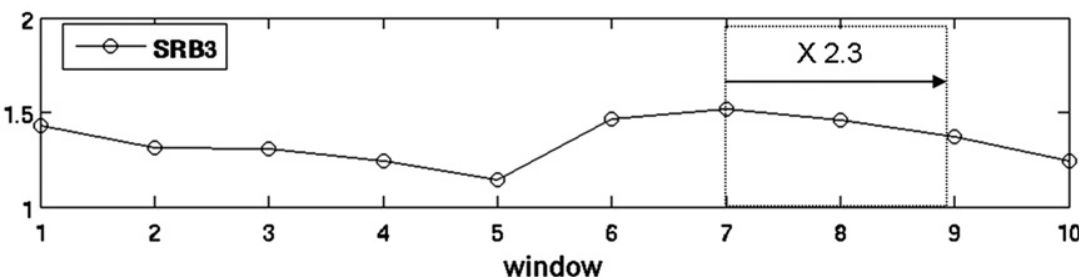

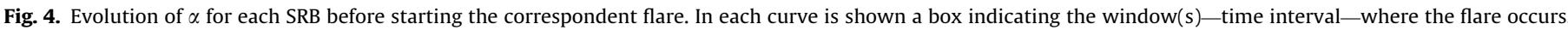

observations of the Sun and the solar-terrestrial environment are required to answer many important questions on long-range correlations among possibly associated energetic events as specific flares, interplanetary CMEs, HSSs and geomagnetic activity. In such a case, many analytical imaging processing techniques are already available for solar physics applications (Aschwanden, 2010). Physically, the behavior of $\alpha$ as shown in Fig. 4 is restricted to the characterization of long-range correlations, due to the underlying turbulent process coming from the non-linear plasma dynamics of the coronal magnetic field. Based only on these measurements one cannot characterize any process on the solarterrestrial magnetic coupling. However, for the events SRB1 and SRB3, from window 5 to window 7 , and from window 6 to window 8 for the event SRB2, it is possible to argue that the persistence of the turbulent process is increasing before the flare occurrence. In order to understand the importance of this behavior into the context of solar active region monitoring, flare forecasting and its interplanetary consequences, it will be necessary to develop further massive analysis considering more specific data as the one explored in this paper. Such data will be available after the Brazilian decimetric array (BDA) becomes operational, what is expected for the beginning of 2012 (see Sawant et al., this issue).

\section{Concluding remarks}

Stochastic intermittent fluctuations are characterized by time series that display multi-scaling, irregular and quasi-regular amplitudes. Usually, intermittency is a characteristic of the underlying dynamics and it is difficult to quantify, since it appears in many variability patterns. Recently, it was shown that decimetric solar bursts observed at $3 \mathrm{GHz}$ due to mutual interacting solar loop with non-linear oscillations require models considering both anisotropy and intermittency. Thus, in the phenomenological analysis of the $3 \mathrm{GHz}$ solar flare considering the scenario given in our previous papers (Rosa et al., 2008), we investigate energy spectra from intermittent MHD turbulent-like stochastic variability patterns with $\beta=2$ (weak turbulence) and $\beta=5 / 3$ (strong turbulence). Here the inhomogeneous nature of the decimetric solar radio emission was successfully detected by using the DFA method on the whole SRB time series. From the point of view of plasma physics, the decimetric SFU time series can be interpreted as being the response of an out-ofequilibrium process, possibly related to the particle acceleration from a transversal loop-loop interaction, where MHD oscillations can play an important role and a counterpart phenomena as turbulent interaction between electron beams and evaporation shocks can act as a secondary source. In order to check such hybrid mechanism composed by an inhomogeneous decimetric extended source, higher spatial resolution data are required. Once the decimetric solar bursts analyzed here are related to geoeffective events, the microphysics processes as particle acceleration and magnetic reconnection can be addressed in a more general scenario involving the plasma solar-terrestrial environment. Then, in the framework of the solar radio activity, our results strongly suggest the use of the DFA scaling exponent as a computational measure for solar radio burst automatic monitoring system (RBAMS), which has been developed for a real-time data system representation (Veronese et al., 2009) in the scope of the Brazilian Space Weather Program.

\section{References}

Alvarez-Ramirez, J., Rodriguez, E., Echeverria, J.C., 2009. A DFA approach for assessing asymmetric correlations. Physica A 388, 2263-2270.

Aschwanden, M.J., Benz, A.O., Dennis, B.R., Schwartz, R.A., 1995. Solar electron beams detected in hard X-rays and radio waves. Astrophysical Journal 455, 347.

Aschwanden, M.J., 2010. Image processing techniques and feature recognition in solar physics. Solar Physics 262 (2), 235-275.

Bai, M.Y.,Zhu, H.B., 2010. Power law and multiscaling properties of the Chinese stock market. Physica A 389, 1883-1890.

Bashan, A., Bartsch, R., Kantelhardt, J.W., Havlin, S., 2008. Comparison of detrending methods for fluctuation analysis. Physica A 387, 5080-5090.

Buldyrev, et al., 1995. Long-range correlation-properties of coding and noncoding DNA sequences-genbank analysis. Physical Review E 51, 5084-5091.

Bunde, A., et al., 2000. Correlated and uncorrelated regions in heart-rate fluctuations during sleep. Physical Review E 85 (17), 3736-3739.

Iyengar, N., Peng, C.-K., Morin, R., Goldberger, A.L., Lipsitz, L.A., 1996. Age-related alterations in the fractal scaling of cardiac interbeat interval dynamics. American Journal of Physiology-Regulatory Integrative and Comparative Physiology 271, R1078-R1084.

Jiricka, K., Karlicky, M., Kepka, O., Tlamicha, A., 1993. Fast drift burst observations with the new Ondrejov radiospectrograph. Solar Physics 147, 203-208.

Kay, S.M., Marple, S.L., 1981. Spectrum analysis—a modern perspective. Proceedings of the IEEE 69 (11), 1380-1419. 
Lobzin, V.V., Cairns, I.H., Robinson, P.A., Steward, G., Patterson, G., 2009. Automatic recognition of type III solar radio bursts: automated radio burst identification system method and first observations. Space Weather 7, S04002.

Miranda, R.A., Chian, A.C.-L., Dasso, S., Echer, E., Munoz, P.R., Trivedi, N.B., Tsurutani, B.T., Yamada, M., 2009. Observation of non-gaussianity and phase synchronization in intermittent magnetic field turbulence in the solar-terrestrial environment. In: Kosovichev, A.G., Andrei, A.H., Rozelot, J.-P. (Eds.), Solar and Stellar Variability: Impact on Earth and Planets, Proceedings IAU Symposium No. 264. Cambridge University Press, pp. 363-368.

Moret, M.A., Zebende, G.F., Nogueira, E., Pereira, M.G., 2003. Fluctuation analysis of stellar X-ray binary systems. Physical Review E 68.

Nindos, A., Aurass, H., Klein, K.-L., Trotter, G., 2008. Radio emission of flares and coronal mass ejections. Solar Physics 253, 3-41.

Osborne, A.R., Provenzale, A., 1989. Finite correlation dimension for stochastic systems with power-law spectra. Physica D 35, 357-381.

Peng C.-K., Buldyrev, S.V., Havlin, S., Simons, M., Stanley, H.E., Goldberger, A.L., 1994. Mosaic organization of DNA nucleotides. Physical Review E 49, 1685-1689.

Rempel, E.L., Chian, A.C.L., Macau, E.E.N., Rosa, R.R., 2004. Analysis of chaotic saddles in low-dimensional dynamical systems: the derivative nonlinear Schrodinger equation. Physica D 199, 407-424.

Rosa, R.R., Karlicky, M., Zanandrea, A., Sych, R.A., Sawant, H.S., Krishan, 2005. Characterization of solar multi-scaling magnetic loop interactions, magnetic fields in the universe: from laboratory and stars to primordial structures. AIP Conference Proceedings 784, 567-573.
Rosa, R.R., Karlický, M., Veronese, T.B., Vijaykumar, N.L., Sawant, H.S., Borgazzi, A.I., Dantas, M.S., Barbosa, E.B.M., Sych, R.A., Mendes, O., 2008. Gradient pattern analysis of solar radio bursts. Advances in Space Research 42, 844-851.

Sawant, H.S., Gopaswamy, N., Rosa, R.R., Sych, R.A., Anfinogentov, S.A., Fernandes, F.C.R., Cecatto, J.R., Costa, J.E., this issue. The Brazilian Decimetric Array and Space Weather.

Sun, W., Dryer, M., Fry, C.D., Deehr, C.S., Smith, Z., Akasofu, S.-I., Kartalev, M.D., Grigorov, K.G., 2002. Real-time forecasting of ICME shock arrivals at L1 during the April Fools Day epoch: 28 March 21 April 2001. Annales Geophysicae 20, 937-945.

Tajima, T., Sakai, J., Nakajima, N., Kosugi, T., Brunel, F., Kundu, M.R., 1987. Current loop coalescence model of solar flares. Astrophysical Journal 321, 1031.

Veronese, T.B., Rosa, R.R., Vijaykumar, N.L., Bolzan, M.J.A., 2009. Generalized numerical lattices for time series representation in complex data systems. Journal of Computational Interdisciplinary Sciences 1 (2).

Watari, S., 1996. Fractal dimensions of the time variation of solar radio emission. Solar Physics 163 (2), 371-381.

Yu, C.X., Gilmore, M., Peebles, W.A., Rhodes, T.L., 2003. Structure function analysis of long-range correlations in plasma turbulence. Physics of Plasmas 10, 2772-2779.

Zaitev, V.V., Kislyakov, A.G., Urpo, S., Stepanov, A.V., Shkelev, E.I., 2003. Spectraltemporal evolution of low-frequency pulsations in the microwave radiation of solar flares. Astronomy Reports 47 (10), 873-882. 